\title{
VALIDATION OF A VIDEO ANALYSIS SOFTWARE PACKAGE FOR QUANTIFYING MOVEMENT VELOCITY IN RESISTANCE EXERCISES
}

Short running head: Validation of video analysis in bench press

Borja Sañudo ${ }^{1}$, David Rueda ${ }^{1}$, Borja del Pozo-Cruz ${ }^{1}$, Moisés de Hoyo ${ }^{1}$, Luis Carrasco ${ }^{1}$

${ }^{1}$ Department of Physical Education and Sport. University of Seville, Seville, Spain.

Disclaimers: none

Source of support: University of Seville

Corresponding Author:

Borja Sañudo, PhD

Physical Education and Sports Department

University of Seville

Pirotécnia s/n, N-41012

Seville

Spain

Tel: $\quad$ ++34 652387090

Email: bsancor@us.es 
Abstract: The aim of this study was to establish the validity of a video analysis software package in measuring mean propulsive velocity (MPV) and the maximal velocity during bench press. Twenty-one healthy males ( $21 \pm 1$ year) with weight training experience were recruited and the MPV and the maximal velocity of the concentric phase ( Vmax) were compared to a linear position transducer system during a standard bench press exercise. Participants performed a one repetition maximum (1RM) test using the supine bench press exercise. The testing procedures involved the simultaneous assessment of bench press propulsive velocity using two kinematic (linear position transducer and semi-auto mated tracking software) systems. High Pearson' correlation coefficients for MPV and Vmax between both devices ( $r=.473$ to .993 ) were observed. The intraclass correlation coefficie nts (ICCS) for barbell velocity data and the kinematic data obtained from video analysis were high ( $>0.79)$. In addition, the low coefficients of variation indicate that measurements had low var iability. Finally, Bland-Altman plots with the limits of agreement of the MPV and Vmax with differ ent loads showed a negative trend which indicated that the video analysis had higher values than the linear transducer. In conclusion, this study has demonstrated that the software employed for the video analysis was an easy to use and cost-effective tool with a very high degree of concurrent validity. This software can be used to evaluate changes in velocity of training load in resistance training which may be important for the prescription and monitoring of training program mes.

Keywords: training intensity; validity; Resistance Training; bench press 


\section{INTRODUCTION}

The control of the training load during resistance training is one of the main concerns for increasing the athletes' performance (9) and both coaches and athletes modify the type of exercise, volume and intensity to get the better results. Exercise intensity is generally recognized to be the most important stimulus associated to changes in strength levels (7). The most commonly used method for assessing the strength training intensity is the percentage of the one repetition maximum (1RM), considered to be the most accurate method to determine maximal dynamic strength. Other approaches to determine exercise intensity include the performance of a given maximal number of repetitions in each set (XRM) or identifying a relative load using repetitions performed to fatigue. However, there are some inherent complications associated with these methods, the $1 \mathrm{RM}$ assessment may be very timeconsuming, it may be associated with injury when performed incorrectly or by novice subjects and is impractical for large groups (9). These limitations encourage the development of new methods to objectively monitor training load during resistance exercise. Many different protocols and devices have been used to assess muscle performance, including the use of isokinetic dynamometry, linear position transducers, accelerometers or force platforms, however, the cost of such equipment, its size, and experimental requirements limit its application to lab-based assessments (5).

Due to the aforementioned limitations there is a need for easy-to-use testing instruments to capture and evaluate athletes' performance at the training site, with real-time feedback to coaches and athletes (16). The analyses of kinematic variables are becoming increasingly accepted for estimating the force and power outputs with exercise (4). Movement velocity is increasingly being used to monitor exercise intensity because movement velocity has a direct relationship with the amount of force exerted $(13,15)$. Movement velocity can also be used as a good estimate of a relative load (\% 1RM), and is additionally a precise indicator of the 
neuromuscular fatigue induced by resistance training (9). However, there has been relatively little discussion in the literature on movement velocity (8). To our knowledge only one previous study has noted that high-speed cameras can be a useful system to provide a realtime velocity tracking (17). Sato et al. (17) in testing a snatch lift found a high correlation between high speed camera and a wireless accelerometer, which has previously been reported to be a valid device for calculating force, velocity and power $(1,10)$.

There is a need for strength and conditioning professionals to visualize workout performances and to track training progress (2), therefore the development of portable, cost-effective equipment that allows kinematic information to be visualized would have obvious advantages in a field-testing situation (5). Any such equipment, however, needs to be valid and reliable. Therefore, in this study we tested the validity of freely available software (Kinovea, version 0.8.15.) that allows semi-automated tracking of objects, specifically we used this software to measure mean velocity in the bench press exercise. Thus, the aim of the current study was to conduct a concurrent validity analysis of the Kinovea software package in measuring mean propulsive velocity (MPV) and the maximal velocity of the concentric phase (Vmax) as compared to a linear position transducer system during bench press in weight-trained males.

\section{METHODS}

\section{Experimental Approach to the Problem}

The intra-session validity of a linear position transducer system and a digitizing software program was calculated when analyzing speed-time variables. Twenty-one subjects performed 1RM testing using the supine bench press exercise. Data were collected simultaneously with a linear position transducer and a digital video camera, MPV and Vmax were determined for each repetition of the bench press. The validity of a freely available digitizing software program in measuring the bench press performance was determined by comparing the MPV measurements with data obtained simultaneously with a linear position transducer. 
Subjects

Twenty-one healthy males with a mean $( \pm S D)$ age, height and body mass of $21 \pm 1$ year, 176.1 $\pm 4.1 \mathrm{~cm}$ and $72.3 \pm 7.0 \mathrm{~kg}$, respectively were recruited. The criteria for study inclusion were a weight training background for a minimum of three years, display a proper technique in bench press and no injuries or conditions that would prevent individuals from safely undertaking the testing procedures. The participants were notified about the potential risks involved and provided their written informed consent. Procedures were approved by the institutional Human Research Ethics Committee.

\section{Procedures}

Participants lay supine on a flat bench, with their feet resting on the bench, and hands placed on the barbell slightly wider than shoulder width. Positions on the bench and grip widths were measured so that they could be individually reproduced on every lift. Participants performed a standardized warm-up consisting of 5 min of pedaling on a cycle ergometer (Kettler Axiom P2, GmbH \& Co.KG, Ense-Parsit, Germany) at a load of 50 W, and upper-body joint mobilization exercises. The warm up was followed by one set of 5 repetitions of bench press with a fixed load of $20 \mathrm{~kg}$. Participants were given instructions on proper lifting techniques and assessment procedures, and were also familiarized with the testing protocols and allowed to practice the tests to eliminate any neurological learning effects. In addition, each subject was carefully instructed to always perform each bench press in an explosive manner, exploding the bar off the chest as fast as possible. A Smith machine (Multipower, Technogym, Spain) was used for all tests.

The testing procedures involved the simultaneous assessment of bench press propulsive velocity using two kinematic (linear position transducer and a semi-automated tracking software) systems. 
A 1RM using the supine bench press exercise was used to assess upper-body strength in each participant. Initial load was set at $20 \mathrm{~kg}$ for all subjects, and was progressively increased in 10 $\mathrm{kg}$ increments until the attained MPV was lower than $0.5 \mathrm{~m} \cdot \mathrm{s}^{-1}$. Thereafter, load was adjusted with smaller increments $(2.5-5 \mathrm{~kg})$. This procedure continued until the participant was not able to complete a single repetition through the full range of motion following the procedures reported elsewhere (15). Rest periods of 2 minutes were allowed between trials. Only the best repetition at each load, according to the criteria of fastest MPV, was considered for analysis.

\section{Data Collection and Analysis}

All testing was performed with the right side of the barbell attached to one linear position transducer (T-Force, T-Force System Ergotech, Murcia, Spain). This system consists of a cableextension linear velocity transducer interfaced to a personal computer by means of a 14-bit resolution analog-to-digital data acquisition board. Vertical instantaneous velocity was sampled at a frequency of $1000 \mathrm{~Hz}$. Simultaneously; a digital video camera (sampling frequency of $50 \mathrm{~Hz}$ ) was placed at $1 \mathrm{~m}$ height at a distance of $2 \mathrm{~m}$ from the Smith machine and aligned perpendicularly to the sagittal plane. The digital images were imported into a freely available digitizing software program (Kinovea 0.8.15, www.kinovea.org). Based on a semiautomatic tracking function, the software allows the calculation of the relevant kinematic parameters of every repetition, providing real time information on screen. In the current study, after the camera set-up was completed, a clear vertical and horizontal reference in the plane of motion was recorded. To minimize the error in the scaling process, the dimensions of the Smith machine were recorded (meters high $\mathrm{x}$ guide bar meters long).

Instantaneous bar velocity was calculated for each time interval as bar displacement over change in time. MPV and Vmax for each movement were obtained for the concentric portion 
of the movement. From T-Force and video data, velocity-time curves were created and Peak values were then obtained for comparison.

\section{Statistical analyses}

Two-dimensional coordinates resulting from the digitizing process (raw data) were then scaled and smoothed using a Butterworth low pass filter with cutoff $6-12 \mathrm{~Hz}$ before velocities were calculated. Paired t-test comparisons were used to compare T-Force and Kinovea results. A regression analysis was performed for testing the linear relationship between the software semi-automated tracking function and the linear transducer mean velocity and mean propulsive velocity values. The goodness of fit and the slope with the $95 \%$ of confidence interval were then calculated. Finally, to test the agreement between both devices, BlandAltman plots were employed. Statistical significance was assumed at $p<0.05$. All analyses were performed using SPSS v.18 (SPSS Inc., Chicago, IL).

\section{RESULTS}

Descriptive statistics for the participants are shown in Table 1. Participants had a BMI of $23.3 \pm$ $2.5 \mathrm{~kg} / \mathrm{m}^{2}$ and displayed a Maximal Propulsive Power (PMP) in the concentric phase of $255.1 \pm$ 74.9 watts.

The analysis of the MPV and Vmax with the linear transducer and the video analysis software for all the different loads are reported in Table 2. The linear relationships of each test with both devices are presented in Table 3, along with the slope (95\% confidence interval $-\mathrm{Cl}-$ ), the 95\% Cls for the limits of agreement from the Bland-Altman analyses and the $y$-intercept values. The relationship for barbell velocity data and the kinematic data obtained from video analysis were high (>0.84) for MPV with the exception of $20 \mathrm{~kg}\left(r^{2}=0.45\right)$ and ranged from $0.41(20 \mathrm{Kg})$ to $0.98(80 \mathrm{Kg})$ for Vmax. In addition, regression plots with the standard regression line of best fit for MPV and Vmax are presented in Figures 1 and 2 respectively. 
Figure 3 shows Bland-Altman plots with the limits of agreement of the MPV with different loads. The bias representing the average difference for measures between both devices was negative which indicate that the video analysis had higher values than the linear transducer. The same trend can be observed for Vmax (Figure 4).

\section{DISCUSSION}

This study was designed to validate measures obtained by kinematic data derived from video analysis in comparison with a linear transducer. The primary finding was that the analysis from the Kinovea software demonstrated high concurrent validity when compared to the laboratory-based instrumentation.

Monitoring the bar velocity during resistance exercise (bench press) is essential since the training effect depends on the velocity at which loads are lifted (8). In the current study, the high correlation coefficients obtained for MPV and Vmax indicate that the data obtained with both methods show a high level of association. The close relationship observed between the MPV in both methods indicate that the video analysis may also allow us to accurately determine the real intensity of effort during exercise (9). Similar values to the current study were reported by Comstock et al. (2) when validating an accelerometer during bench press. It is important to note that lower correlation coefficients were obtained with $20 \mathrm{Kg}(r=.676$ and $r=.473)$ and $30 \mathrm{Kg}(r=.921$ and $r=.594)$ for MPV and Vmax respectively, but correlation were better with the heavier loads tested, which is consistent with the results observed by Crewther et al. (4). One possible explanation for these results may be technique modifications adopted by participants with low loads (6), this may lead to the use of different muscles and/or biomechanics during the bench press.

Despite the good correlation between the two methods, the $95 \% \mathrm{Cls}$ for the slope values from the regressions and the t-tests from the mean comparisons may indicate an over-prediction of the video analyses relative to the transducer. In addition, caution exercised in interpreting the 
data as horizontal movements of the bar away from the vertical vector, especially during freeweight exercises, can modify the results and may disrupt the accuracy of velocity assessment. However, in the current study these issues were eliminated by using the Smith machine. Although it is beyond the scope of the current study, previous studies demonstrated that a change in horizontal displacement of a weight bar may result in overestimations of vertical velocity (3). However, with the auto-tracking module of the Kinovea software these deviations can be identified and can be minimized. Future studies are warranted to address the validity of each kinematic system during exercise performed in multiple planes (4).

Another interesting finding of the present study is that high regression coefficients also indicate a high agreement between both devices (11). Together with the Bland-Altman analysis it can be confirmed the good consistency, as results from this analysis showed that systematic errors (mean difference between devices) for the kinematics outcomes assessed were nearly zero and the $95 \%$ limits of agreement were narrow. However it is important to note that the data obtained from the video analysis overestimated the velocity. Therefore, while the two methods are correlated with each other, the agreement between the measures can be considered as modest. The different velocities of data collection (linear transducer; $1000 \mathrm{~Hz}$ compared to video analysis; $50 \mathrm{~Hz}$ ) may explain these variations.

There are some limitations when using the software for video analysis. First, there is a need to standardize the movement as data are directionally dependent and, as stated earlier, it is usually recommended that the movement is performed on a Smith machine. The Smith machine which eliminate sagittal movement of the barbell during the test. This source of error can also be related to the experience of the lifters. Further study would be necessary to determine the influence of these issues on the kinematic variables measured. Second, it should be noted that possible differences in the technical characteristics of both devices (e.g. sampling rate) may lead to different results. 
Despite these limitations, the semi-automatic tracking function incorporated in the freely available Kinovea software, demonstrates a high degree of concurrent validity as a field testing instrument. The use of this instrument is appropriate to evaluate changes in velocity for the bench press exercise and therefore it may also be used to assess and monitor changes in strength performance.

\section{PRACTICAL APPLICATIONS}

The findings of this study have important practical applications for the prescription and monitoring of training load in resistance training. The use of velocity measuring devices during training is often limited to laboratory-type assessment; however, this software will offer a portable, cost-effective technique for the assessment of training. The use of velocity as a measure allows selecting the appropriate weight for athletes, this tool will let the coach give online support services and evaluate an athlete's strength without the need to perform a 1RM test. Several authors highlighted that with accelerometers it was possible to modify the exercise prescriptions in real time and thus evaluate training progression $(12,14)$ and in our opinion, these benefits can also be attributable to this software. Therefore, based on the ease of use, the cost effectiveness and the possibilities of remote support, our results underline the practical importance of considering video analysis (with the Kinovea software auto-tracking tool) for monitoring the training load in resistance exercises. 


\section{REFERENCES}

1. Casartelli, N, Muller, R, and Maffiuletti, NA. Validity and reliability of the Myotest accelerometric system for the assessment of vertical jump height. J Strength Cond Res 24: $3186-3193,2010$.

2. Comstock, BA, Solomon-Hill, G, Flanagan, SD, Earp, JE, Luk, HY, Dobbins, KA, Dunn-Lewis, C, Fragala, MS, Ho, JY, Hatfield, DL, Vingren, JL, Denegar, CR, Volek, JS, Kupchak, BR, Maresh, CM, and Kraemer, WJ. Validity of the Myotest ${ }^{\circledR}$ in measuring force and power production in the squat and bench press. J Strength Cond Res 25: 2293-7, 2011.

3. Cormie, P, Deane, R, and McBride, JM. Methodological concerns for determining power output in the jump squat. J Strength Cond Res 2007; 21: 424-430.

4. Crewther, BT, Kilduff, LP, Cunningham, DJ, Cook, C, Owen, N, and Yang, GZ. Validating two systems for estimating force and power. Int J Sports Med 32: 254-8, 2011.

5. Cronin, JB, Hing, RD, and McNair, PJ. Reliability and validity of a linear position transducer for measuring jump performance. J Strength Cond Res 18: 590-593, 2004.

6. Cronin, JB, McNair, PJ, and Marshall, RN. Force-velocity analysis of strength-training techniques and load: implications for training strategy and research. J Strength Cond Res 17: 148-55, 2003.

7. Fry, AC. The role of resistance exercise intensity on muscle fibre adaptations. Sports Med 34: $663-679,2004$.

8. González-Badillo, JJ, Marques, MC, and Sánchez-Medina, L. The importance of movement velocity as a measure to control resistance training intensity. J Hum Kinet 29A: 15-9, 2011.

9. González-Badillo, JJ, and Sánchez-Medina, L. Movement velocity as a measure of loading intensity in resistance training. Int J Sports Med 31: 347-52, 2010.

10. Jidovtseff, B, Crielaard, JM, Cauchy, S, and Croisier, JL. Validity and reliability of an inertial dynamometer using accelerometry. Sci Sport 23: $94-97,2008$. 
11. Knudson, DV. Significant and meaningful effects in sports biomechanics research. Sport Biomech 8: 96-104, 2009.

12. Kraemer, WJ and Fleck, SJ. Optimizing Strength Training: Designing Nonlinear Periodization Workouts. Champaign, IL: Human Kinetics, 2007.

13. Miletello, WM, Beam, JR, and Cooper, ZC. A biomechanical analysis of the squat between competitive collegiate, competitive high school, and novice powerlifters. J Strength Cond Res 23: 1611-1617, 2009.

14. Rucci, JA and Tomporowski, PD. Three types of kinematic feedback and the execution of the hang power clean. J Strength Cond Res 24: 771-778, 2010.

15. Sanchez-Medina, L, Perez, CE, and Gonzalez-Badillo, JJ. Importance of the propulsive phase in strength assessment. Int J Sports Med 31: 123-9, 2010.

16. Sato, K, Sands, WA, and Stone, MH. The reliability of accelerometry to measure weightlifting performance. Sports Biomech 11: 524-31, 2012.

17. Sato, K, Smith, SL, and Sands, WA. Validation of accelerometer for measuring sport performance. J Strength Cond Res 23: 341-347, 2009. 
FIGURE LEGENDS

Figure 1. Regression plots for MPV

Figure 2. Regression plots for Vmax

Figure 3. Bland-Altman Plots with limits of agreement of MPV

Figure 4. Bland-Altman Plots with limits of agreement of Vmax

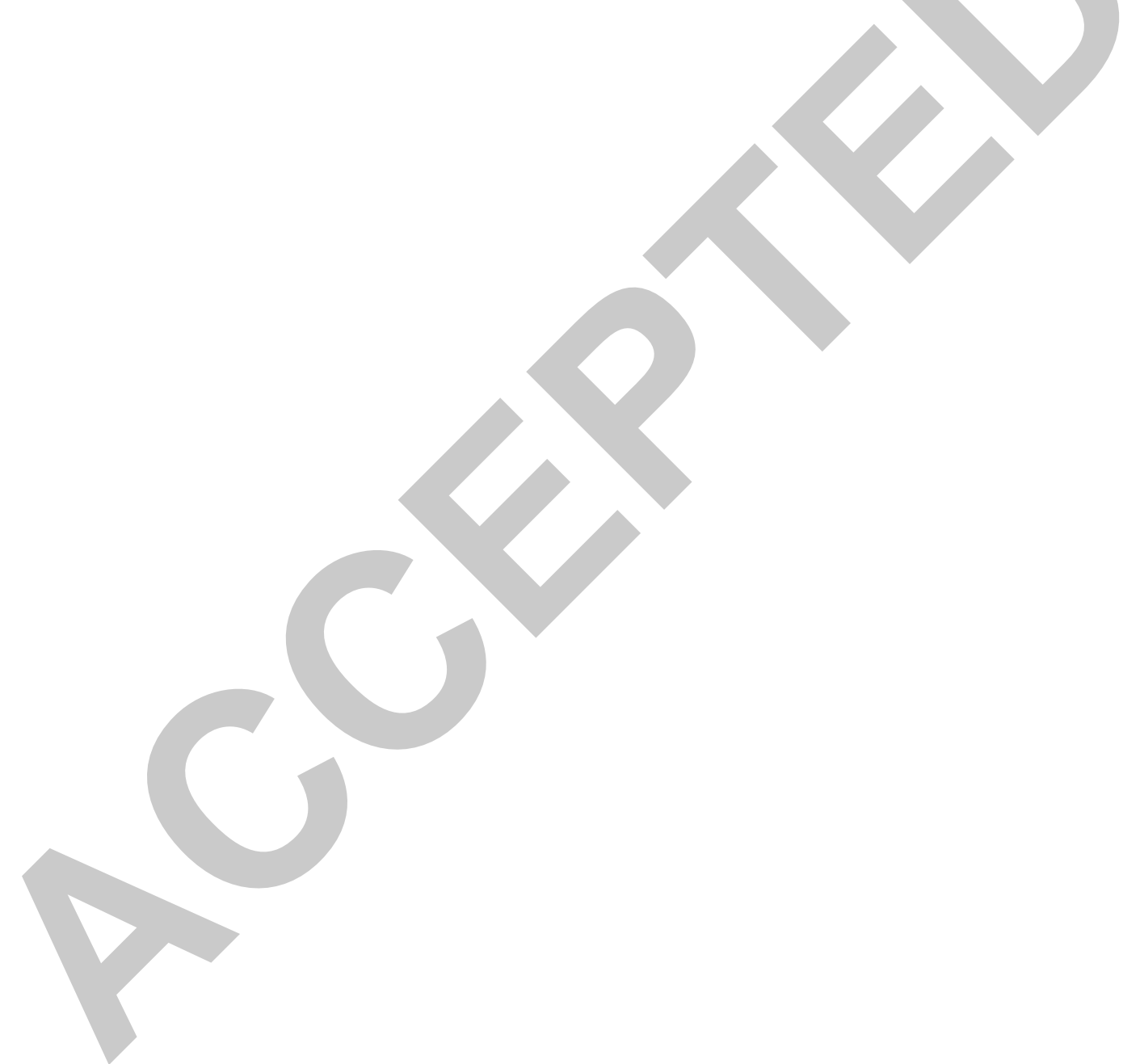


Table 1. Descriptive analysis of the participants $(n=21)$

\begin{tabular}{lccc} 
Outcome & Mean & SD & Range \\
Age (years) & 21 & 1 & $20-22$ \\
Heigh (cm) & 176.18 & 4.03 & $168-182$ \\
Weigh (kg) & 72.31 & 7.02 & $61.3-84.2$ \\
BMI (kg/m ${ }^{2}$ ) & 23.33 & 2.50 & $20.1-28.9$ \\
PMP (Watts) & 255.06 & 74.93 & $158.9-397.3$ \\
\hline BMI: Body Mass Index; PMP: Maximal Propulsive Power
\end{tabular}


Table 2. Comparative analysis of kinematics data obtained with the linear transducer and the software at different loads

T-Force

Kinovea

\begin{tabular}{|c|c|c|c|c|c|c|c|c|}
\hline \multirow[b]{2}{*}{ Load } & \multirow[b]{2}{*}{ Outcome } & \multirow[b]{2}{*}{ Mean } & \multicolumn{6}{|c|}{ Mean difference } \\
\hline & & & SD & Mean & SD & (95\% CIs) & $t$ & $P$ \\
\hline \multirow{2}{*}{$20 \mathrm{Kg}$} & $\operatorname{MPV}\left(\mathrm{m} \mathrm{s}^{-1}\right)$ & 1.05 & 0.13 & 1.48 & 0.18 & $-0.43(-0.49$ to -0.37$)$ & -15.067 & $<.001$ \\
\hline & $\operatorname{VMAX}\left(\mathrm{m} \mathrm{s}^{-1}\right)$ & 1.64 & 0.20 & 2.22 & 0.32 & $-0.57(-0.70$ to -0.44$)$ & -9.164 & $<.001$ \\
\hline \multirow{2}{*}{$30 \mathrm{Kg}$} & $\operatorname{MPV}\left(\mathrm{m} \mathrm{s}^{-1}\right)$ & 0.89 & 0.14 & 1.30 & 0.16 & $-0.41(-0.43$ to -0.38$)$ & -30.357 & $<.001$ \\
\hline & $\operatorname{VMAX}\left(\mathrm{m} \mathrm{s}^{-1}\right)$ & 1.33 & 0.18 & 1.92 & 0.27 & $-0.59(-0.68$ to -0.49$)$ & -12.437 & $<.001$ \\
\hline \multirow{2}{*}{$40 \mathrm{Kg}$} & $\operatorname{MPV}\left(\mathrm{m} \mathrm{s}^{-1}\right)$ & 0.71 & 0.15 & 1.02 & 0.19 & $-0.30(-0.33$ to -0.28$)$ & -27.324 & $<.001$ \\
\hline & $\operatorname{VMAX}\left(\mathrm{m} \mathrm{s}^{-1}\right)$ & 1.11 & 0.19 & 1.53 & 0.29 & $-0.42(-0.48$ to -0.36$)$ & -14.824 & $<.001$ \\
\hline \multirow{2}{*}{$50 \mathrm{Kg}$} & $\operatorname{MPV}\left(\mathrm{m} \mathrm{s}^{-1}\right)$ & 0.53 & 0.15 & 0.76 & 0.21 & $-0.23(-0.26$ to -0.21$)$ & -18.135 & $<.001$ \\
\hline & $\operatorname{VMAX}\left(\mathrm{m} \mathrm{s}^{-1}\right)$ & 0.88 & 0.19 & 1.24 & 0.26 & $-0.36(-0.40$ to -0.32$)$ & -18.506 & $<.001$ \\
\hline \multirow{2}{*}{$60 \mathrm{Kg}$} & $\operatorname{MPV}\left(\mathrm{m} \mathrm{s}^{-1}\right)$ & 0.38 & 0.12 & 0.54 & 0.17 & $-0.16(-0.18$ to -0.13$)$ & -14.574 & $<.001$ \\
\hline & $\operatorname{VMAX}\left(\mathrm{m} \mathrm{s}^{-1}\right)$ & 0.71 & 0.15 & 0.99 & 0.22 & $-0.28(-0.32$ to -0.23$)$ & -12.849 & $<.001$ \\
\hline \multirow{2}{*}{$70 \mathrm{Kg}$} & $\operatorname{MPV}\left(\mathrm{m} \mathrm{s}^{-1}\right)$ & 0.32 & 0.13 & 0.47 & 0.18 & $-0.14(-0.21$ to -0.08$)$ & -5.310 & .002 \\
\hline & $\operatorname{VMAX}\left(\mathrm{m} \mathrm{s}^{-1}\right)$ & 0.65 & & 0.93 & 0.32 & $-0.28(-0.45$ to -0.10$)$ & -3.939 & .008 \\
\hline \multirow{2}{*}{$80 \mathrm{Kg}$} & $\operatorname{MPV}\left(\mathrm{m} \mathrm{s}^{-1}\right)$ & 0.31 & 0.11 & 0.47 & 0.19 & $-0.16(-0.34$ to 0.02$)$ & -3.769 & .064 \\
\hline & $\operatorname{VMAX}\left(\mathrm{m} \mathrm{s}^{-1}\right)$ & 0.61 & 0.19 & 0.84 & 0.24 & $-0.23(-0.36$ to -0.10$)$ & -7.876 & .016 \\
\hline
\end{tabular}

MPV: mean propulsive velocity; VMAX: maximal velocity of the concentric phase; $r$ : Pearson product-

moment correlation coefficients 
Table 3. Linear relationship between the MVP and Vmax values as measured by video analysis and the linear transducer.

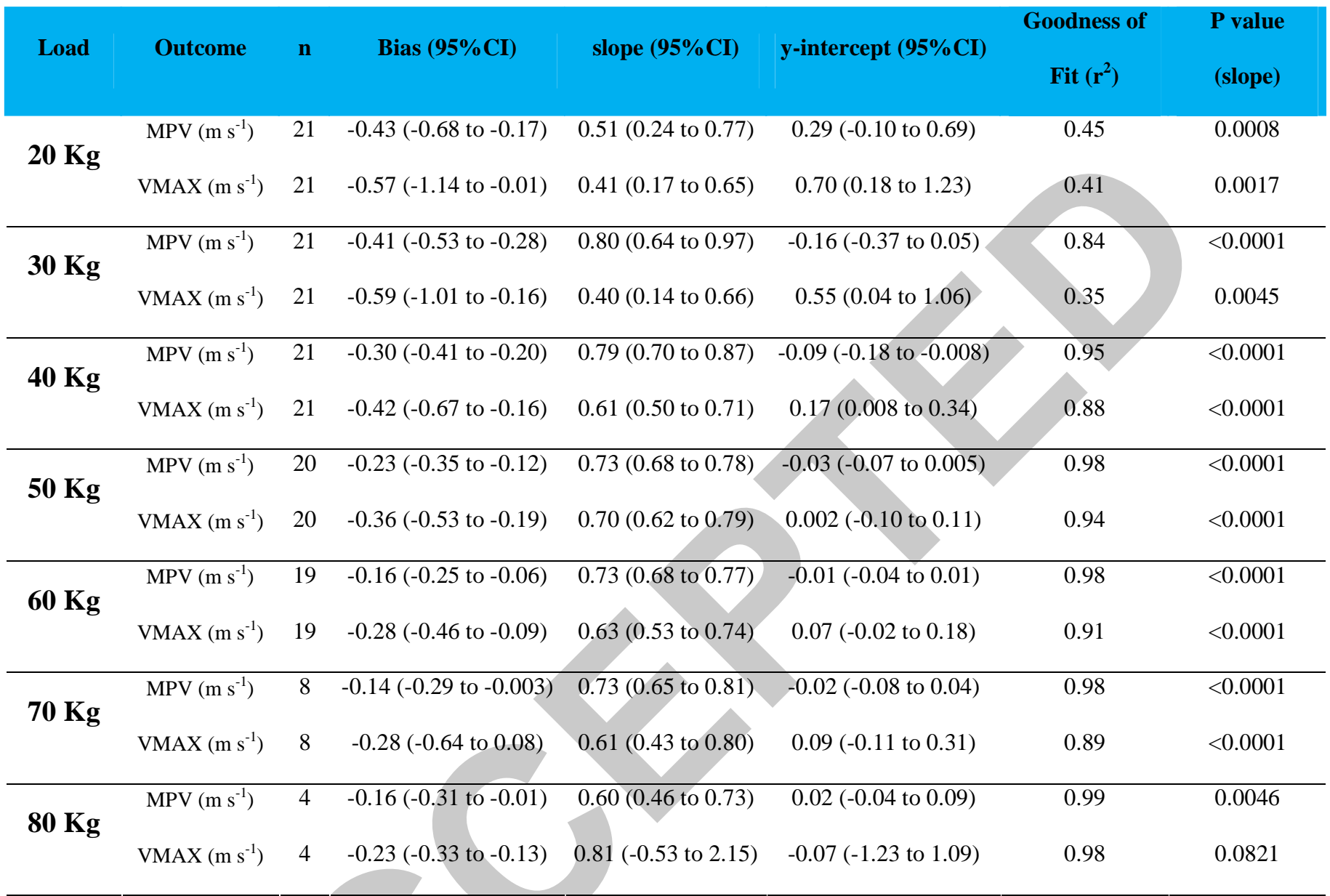

MPV: mean propulsive velocity; VMAX: maximal velocity of the concentric phase; 
Mean Propulsive Velocity $(20 \mathrm{~kg}$ )

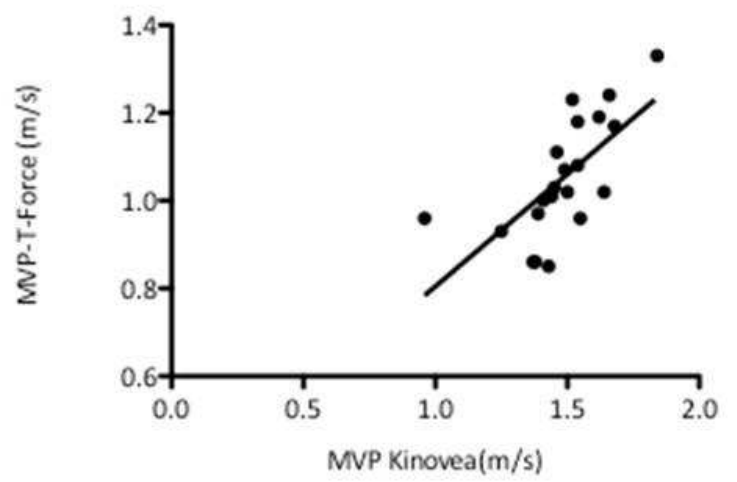

Mean Propulsive Velocity $(40 \mathrm{~kg})$

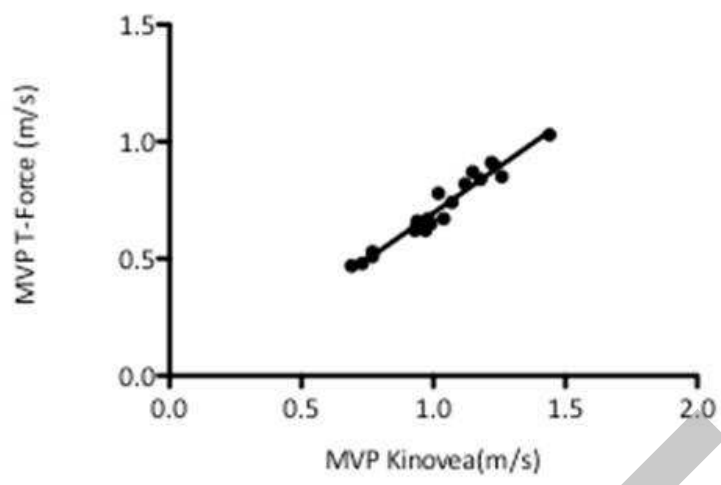

Mean Propulsive Velocity (60kg)

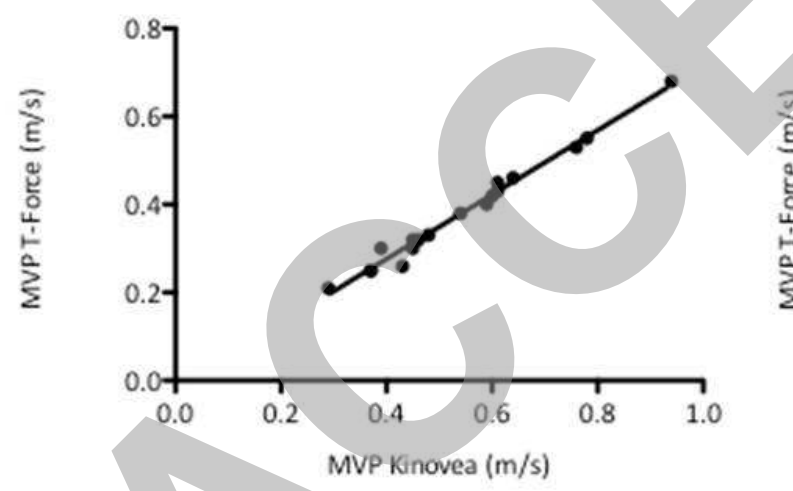

Mean Propulsive Velocity (30kg)
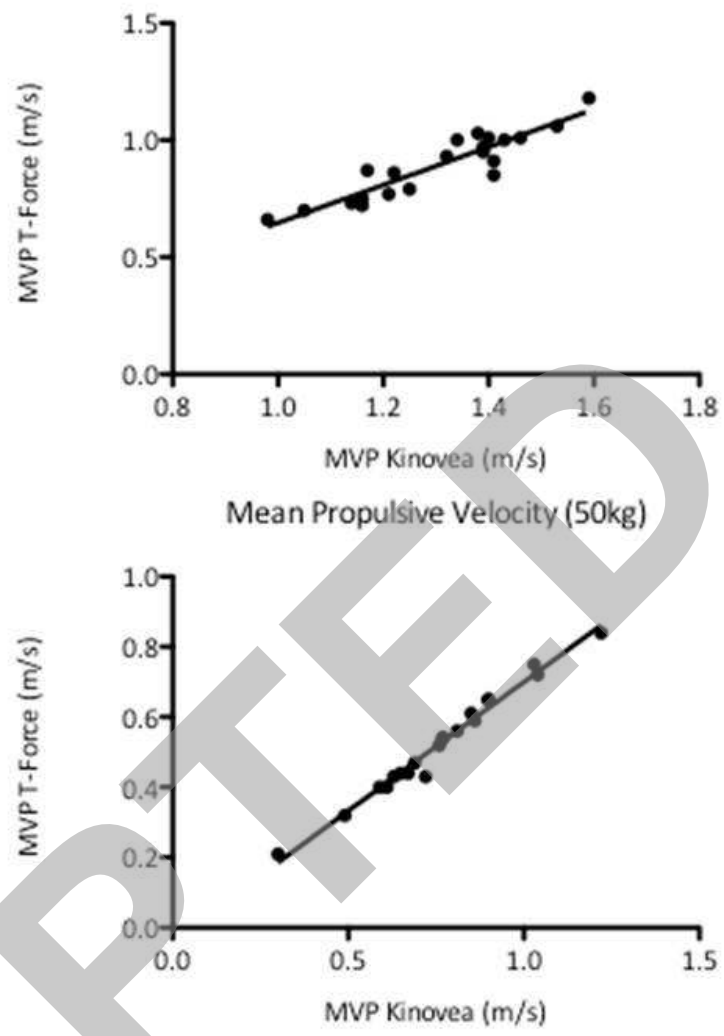

Mean Propulsive Velocity $(70 \mathrm{~kg})$

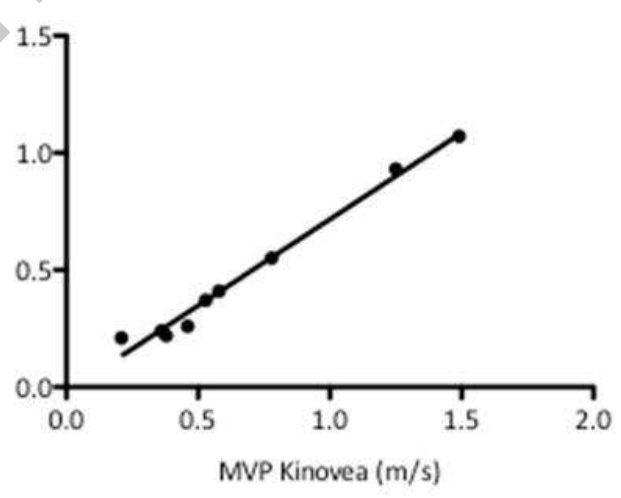


Maximum Velocity $(20 \mathrm{~kg})$

Maximum Velocity $(30 \mathrm{~kg})$
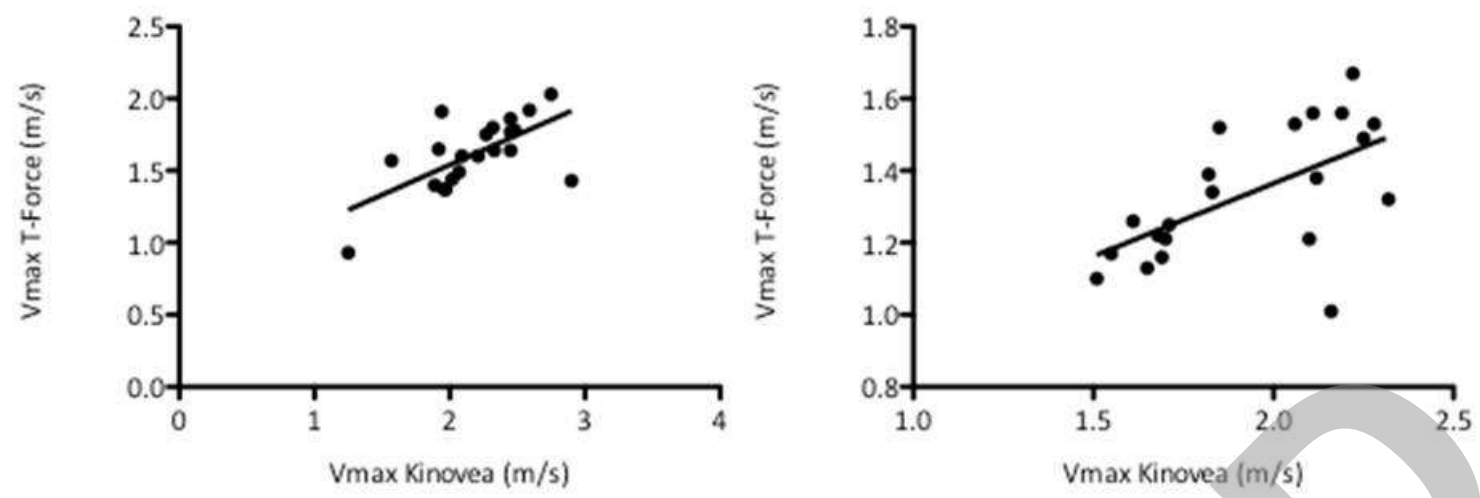

Maximum Velocity $(40 \mathrm{~kg})$

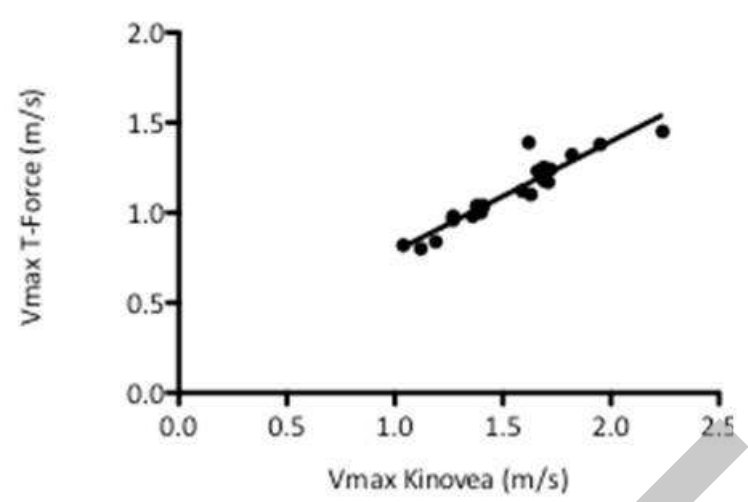

Maximum Velocity $(50 \mathrm{~kg})$

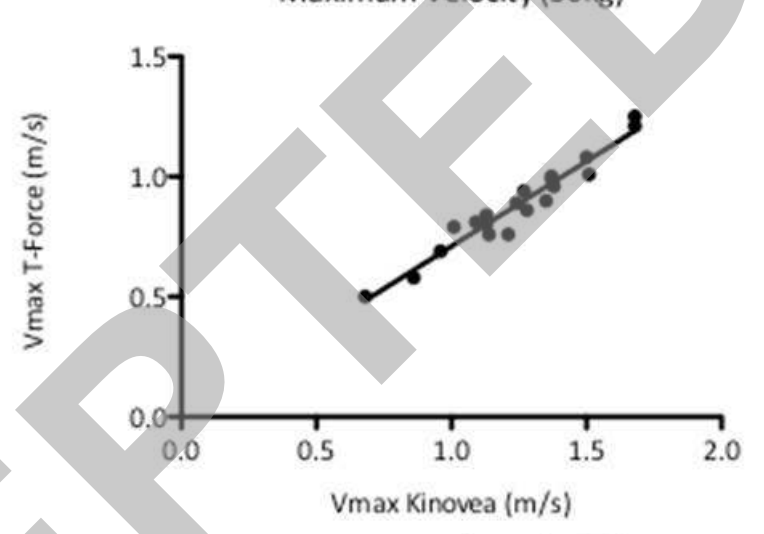

Maximum Velocity $(60 \mathrm{~kg})$

Maximum Velocity $(70 \mathrm{~kg})$
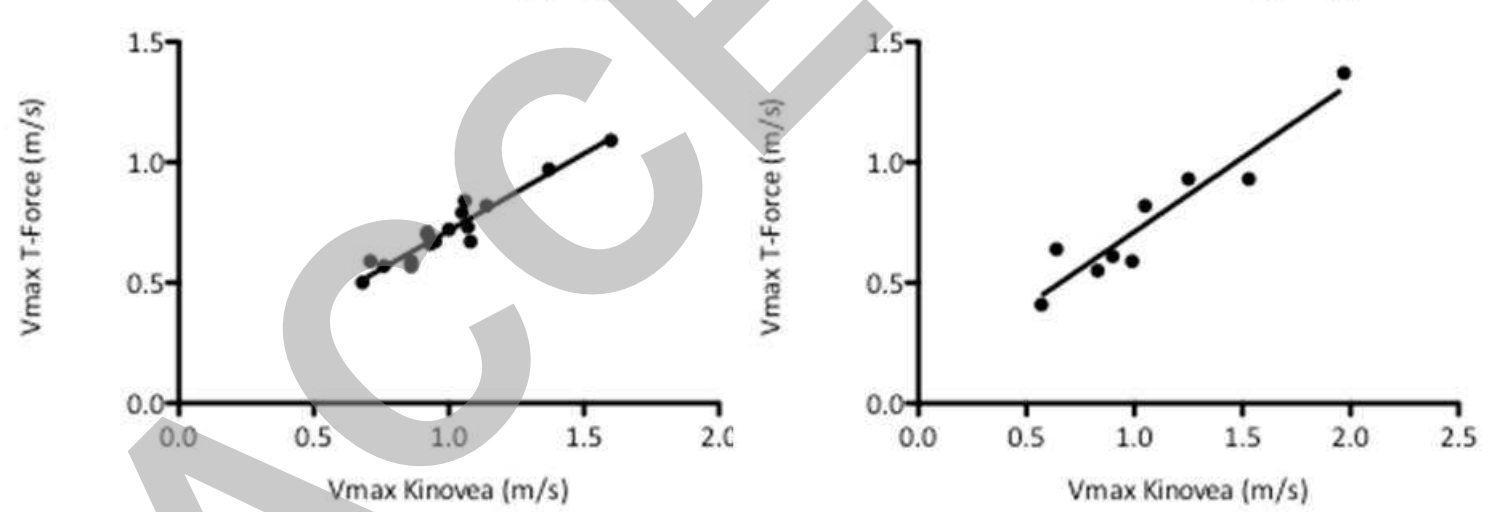
Mean Propulsive Velocity $(20 \mathrm{~kg})$

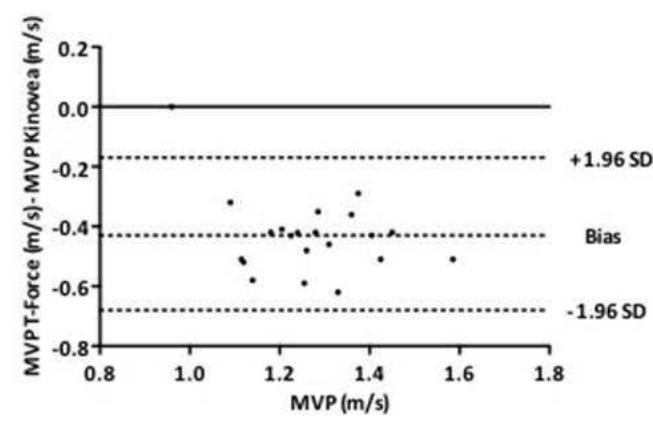

Mean Propulsive Velocity (40 kg)

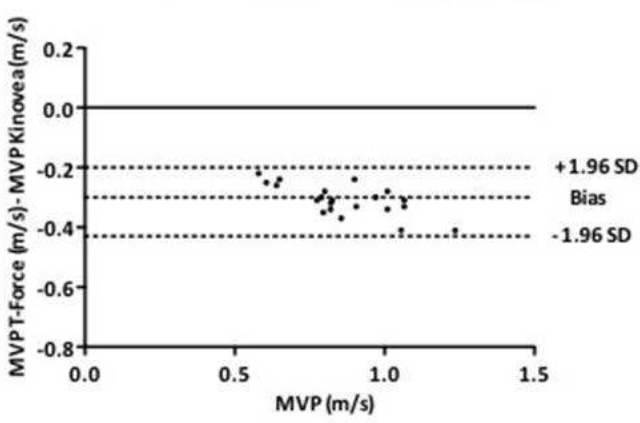

Mean Propulsive Velocity $(60 \mathrm{~kg})$

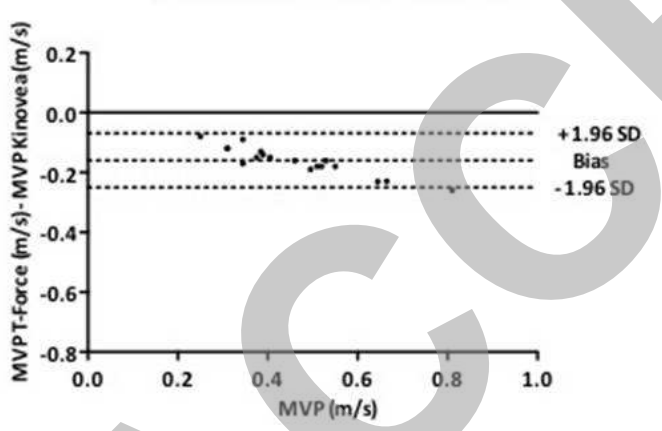

Mean Propulsive Velocity (30 kg)

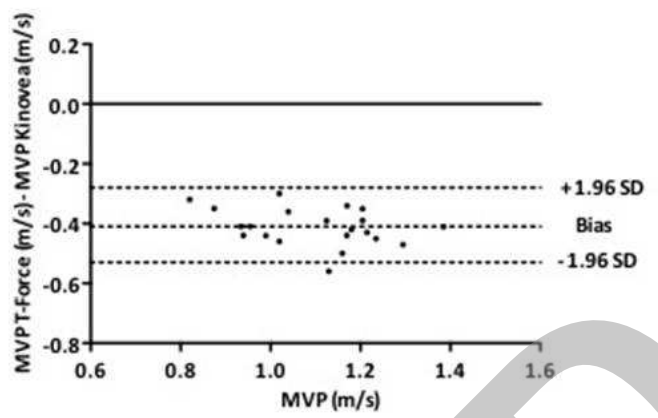

Mean Propulsive Velocity (50 kg)

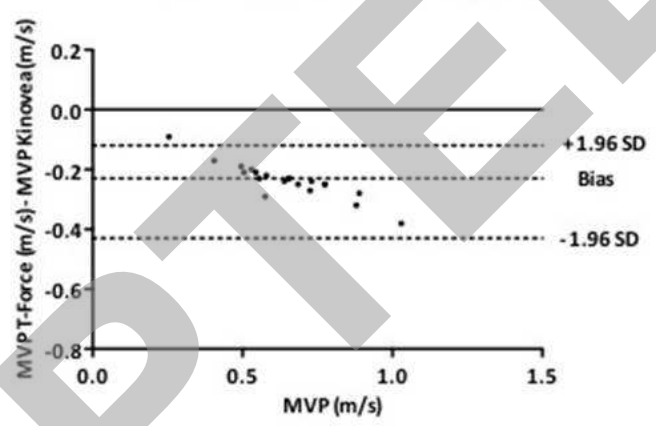

Mean Propulsive Velocity $(70 \mathrm{~kg})$

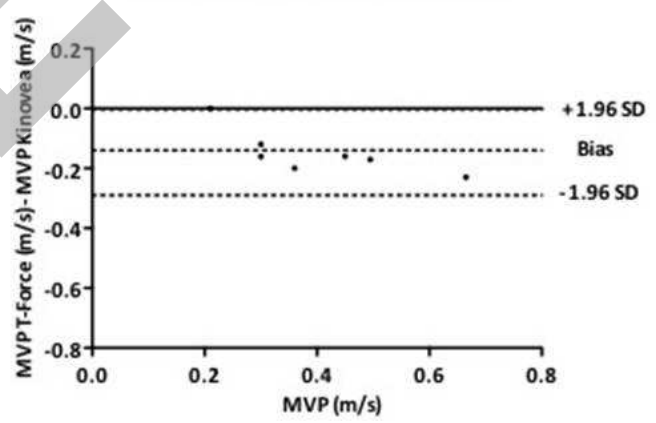



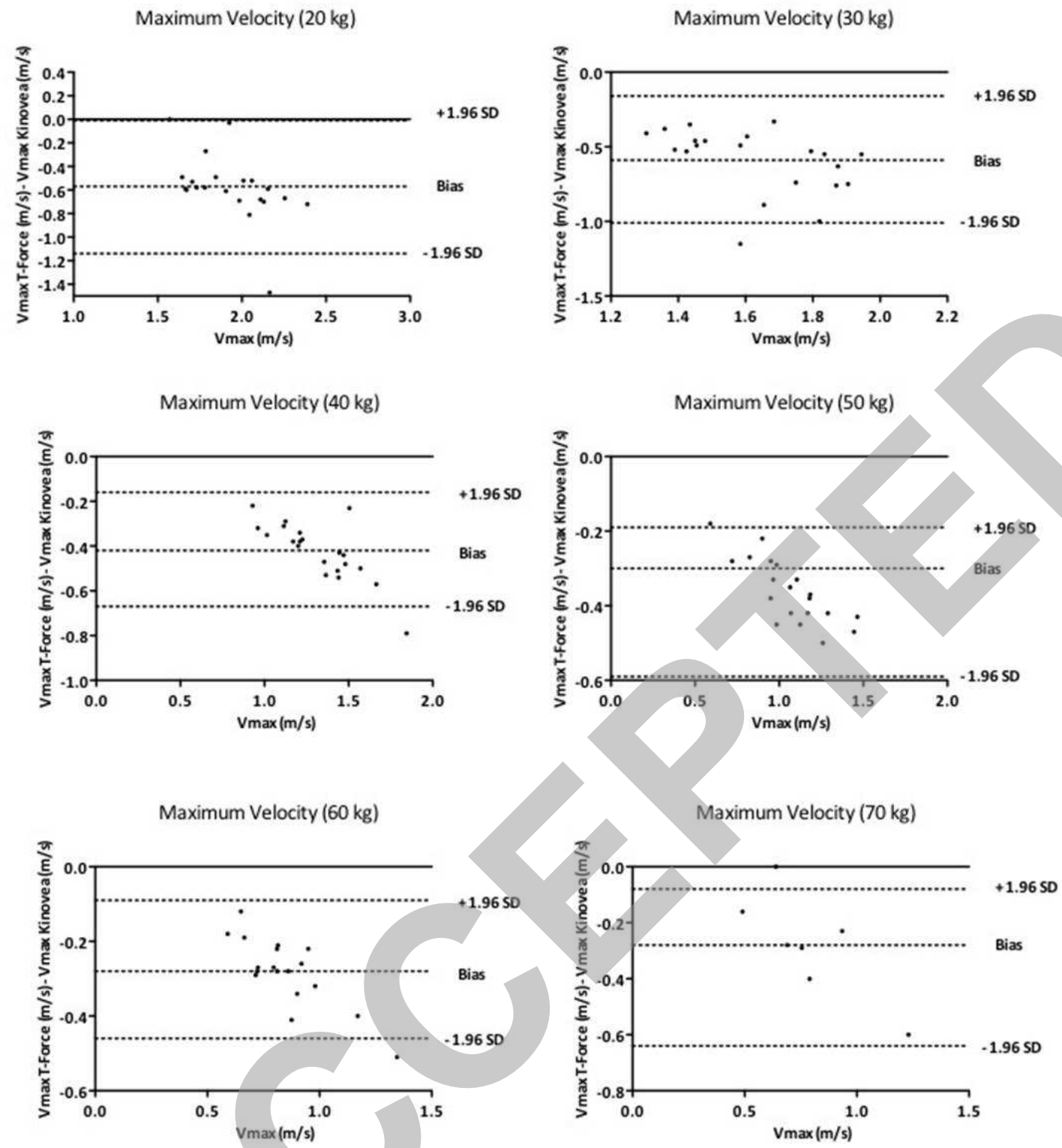